\title{
Avaliação de produção científica de pesquisadores: mapeamento das publicações científicas
}

Evaluation researcher' scientific production: mapping the scientific publications

\section{Cleomir Waiczyk}

Administrador pela Faculdade Cenecista de Joinville

Mestrando em Engenharia de Produção pela Universidade Federal de Santa Catarina

Endereço: Rua Babitonga, ${ }^{\circ} 515$ - Bairro Floresta

CEP: 89210-040 - Joinville/SC - Brasil

E-mail:w.cleomir@gmail.com

Telefone: (47) 9658-4876

\section{Eduardo Rolim Ensslin}

Mestre em Engenharia de Produção pela Universidade Federal de Santa Catarina

Pesquisador Autônomo

Endereço: Rua Júlio de Castilhos, n॰ 1760, Bairro Centro

CEP: 96.300-000 - Jaguarão/RS - Brasil

E-mail: eduardo_ensslin@hotmail.com

Telefone: (53) 9122-5899

Artigo recebido em 01/02/2013. Revisado por pares em 23/04/2013. Reformulado em 20/05/2013. Recomendado para publicação em 14/06/2013 por Sandra Rolim Ensslin (Editora Científica). Publicado em 09/08/2013. 


\title{
Resumo
}

O aumento da produção científica, aliado à evolução tecnológica, gerou uma quantidade de publicações que é incompatível com os processos tradicionais de busca e seleção de materiais para dar suporte à pesquisa. Assim, esta pesquisa objetivou identificar, por meio do instrumento ProKnow-C, artigos com reconhecimento científico e alinhados ao tema sobre avaliação da produção científica de pesquisadores disponíveis na literatura internacional e realizar uma bibliometria. Como resultado, identificou-se 15 artigos para compor o Portfólio Bibliográfico, onde se evidenciou: "Scientometrics" como periódico mais presente; "An index to quantify an individual's scientific research output" como artigo mais citado e van Raan como autor de destaque.

Palavras-chave: Avaliação de desempenho. ProKnow-C. Produção científica. Avaliação docente. Artigos científicos.

\begin{abstract}
The growth in scientific production, together with technological evolution, has generated a number of publications that is inconsistent with the traditional search methods and selection of materials to support research. Thus, this study aimed at identifying, through the ProKnow$C$ tool, articles that were scientifically recognized and aligned to the topic "Evaluation of Scientific Production of Researchers" (available in the international literature), and to conduct bibliometrics with such material. As a result, 15 articles were identified to compose the Bibliographical Portfolio, in which it was observed that the Scientometrics was the journal that appeared the most on the search; An index to quantify an individual's scientific research output was the most cited article, and van Raan the prominent author.
\end{abstract}

Keywords: Performance Evaluation. ProKnow-C. Scientific production. Teacher assessment. Scientific articles.

\section{Introdução}

A carreira do magistério, nas instituições de Ensino Superior de elite no Brasil nas últimas décadas, passou a ter como um de seus principais fatores de diferenciais de qualidade as publicações. Assim, a avaliação de produções científicas passou a receber atenção especial. Como esse atributo foi reconhecido internacionalmente como fator de importância, a maioria dos pesquisadores passou a desenvolver pesquisas e a submeter aos periódicos existentes. Isso fez com que o número de artigos, periódicos e bancos de dados crescessem de tal maneira que hoje a quantidade de trabalhos publicados excede a capacidade de busca e seleção dos pesquisadores.

Tal contexto motivou a criação de uma área da ciência da informação, a Cientometria, que consiste em trazer para o leitor informações quantitativas sobre variáveis de destaque nas publicações em determinado tema. Essa temática estimulou os presentes pesquisadores a realizarem uma bibliometria referente a materiais que envolvam a Cientometria para explicitar o que tem sido realizado no assunto da avaliação do desempenho de pesquisadores. 
A partir desse contexto, surge a seguinte indagação da pesquisa: como encontrar e selecionar as publicações e suas respectivas variáveis de destaque sobre o tema Cientometria por meio de um processo estruturado?

Assim, o objetivo geral desta pesquisa é identificar artigos disponíveis na literatura internacional que abordam sistemas de avaliação de produções científicas e, para esses artigos, realizar uma bibliometria. Para tanto, foram definidos os seguintes objetivos específicos: (i) selecionar um portfólio bibliográfico com reconhecimento científico e alinhado ao tema, segundo a percepção dos pesquisadores; e (ii) analisar quantitativamente os periódicos, artigos, autores e palavras-chave do portfólio bibliográfico.

Como instrumento de intervenção, utilizar-se-á o método Knowledge Development Process - Constructivist (ProKnow-C), conforme os trabalhos desenvolvidos por Ensslin et al. (2010), Tasca et al. (2010), Lacerda, Ensslin e Ensslin (2011), Ensslin, Ensslin e Pacheco (2012), Chaves et al. (2012) e Rosa et al. (2012).

A pesquisa evidenciará um processo estruturado para lidar com temas atuais a fim de desenvolver o conhecimento, nos pesquisadores, requerido para iniciar a pesquisa de um tema e o ilustrará por meio do tema "Avaliação das Publicações Científicas de Pesquisadores". Tal conhecimento será, então, compartilhado com a comunidade científica neste trabalho.

Este artigo está constituído por cinco seções. Além dessa introdução, a metodologia de pesquisa será apresentada na segunda seção. Na terceira seção, será exposto o referencial teórico. Em seguida, na quarta seção, serão abordados os resultados e discussões. Por fim, serão apontadas as considerações finais.

\section{Metodologia da Pesquisa}

Esta seção está dividida em duas subseções: enquadramento metodológico e instrumento de intervenção.

\subsection{Enquadramento Metodológico}

A presente pesquisa é de natureza exploratório-descritiva. É exploratória por proporcionar uma investigação sobre um tema específico, bem como uma avaliação de desempenho de produção científica, permitindo uma visão mais ampla sobre o assunto e o esclarecendo por intermédio deste trabalho; e descritiva, por permitir estudar as características de um determinado grupo de variáveis (RICHARDSON, 2008).

Quanto à natureza da pesquisa, é denominada como teórico-ilustrativa. Pois, tem o objetivo de ilustrar a operacionalização do instrumento ProKnow-C, para busca e análise bibliométrica de artigos sobre o tema da avaliação da produção científica de pesquisadores, a fim de que outros pesquisadores possam fazer uso desse instrumento e, assim, conseguir selecionar um portfólio bibliográfico relevante e alinhado ao tema de pesquisa que estiverem investigando (FERREIRA; YOSHIDA, 2004).

A lógica da pesquisa é indutiva por realizar a observação de informações de um problema cujo conhecimento se pretende adquirir e explicitá-lo durante o decorrer do trabalho (GIL, 2008).

Os dados foram coletados de forma primária, originários das delimitações postas pelos pesquisadores para o processo de seleção das publicações a fim de compor o portfólio

99 ISSN 2175-8069, UFSC, Florianópolis, v. 10, n. 20, p. 97-112, mai./ago. 2013 
bibliográfico por meio de abordagem qualitativa; e de forma secundária, quando os artigos selecionados, assim como as informações relacionadas, foram extraídos de fonte comuns à comunidade científica. Ao analisar os periódicos, artigos, autores e palavras-chave, adotou-se uma abordagem quantitativa (RICHARDSON, 2008).

Os resultados da pesquisa se caracterizam como aplicada, permitindo que o conhecimento adquirido seja utilizado para a realização dos objetivos específicos (RICHARDSON, 2008). Adotou-se a pesquisa bibliográfica como procedimento técnico pelo fato de compor um arcabouço teórico (GIL, 2008).

\subsection{Instrumento de Intervenção}

Dada a importância de se buscarem alternativas teóricas e metodologias que possibilitem mapear as informações requeridas para a pesquisa científica pertinente ao campo, a profundidade teórica e o rigor metodológico de forma confiável (KOBASHI; SANTOS, 2006), faz-se necessária a utilização de um processo estruturado que englobe aspectos considerados relevantes pelos pesquisadores.

Dessa forma, o instrumento de intervenção utilizado nesta pesquisa é o Knowledge Development Process - Constructivist (ProKnow-C), encontrado nos trabalhos de Tasca et al. (2010), Lacerda, Ensslin e Ensslin (2011) e Rosa et al. (2012), por permitir atingir os objetivos e delimitações postas pelos pesquisadores para a seleção de portfólio bibliográfico e análise bibliométrica de forma estruturada e gradual.

O ProKnow-C teve sua origem no Laboratório de Metodologia Multicritério de Apoio à Decisão (LabMCDA), do Departamento de Engenharia de Produção e Sistemas da Universidade Federal de Santa Catarina, Brasil, que investiga, desde 1994, o tema "Avaliação de Desempenho Organizacional como Instrumento de Apoio à Decisão", com ênfase na metodologia Multicritério de Apoio à Decisão - Construtivista (TASCA et al., 2010; AFONSO et al., 2012; ENSSLIN; ENSSLIN; PACHECO, 2012).

Após o laboratório identificar a ausência de um processo com o propósito de fornecer o suporte necessário para selecionar artigos relevantes para compor um portfólio bibliográfico, iniciou-se, a partir de 2005, a implementação de uma linha de pesquisa que pudesse desenvolver esse processo e que atendesse, de forma estruturada, à amplitude, às delimitações e ao foco orientado no assunto definido pelo pesquisador (TASCA et al., 2010; LACERDA; ENSSLIN; ENSSLIN, 2011; ROSA et al., 2012).

Em 2007 e 2008, surgiram as primeiras versões do processo que, em 2011, viria a receber o nome de ProKnow-C, tendo suas primeiras publicações já no ano anterior e sua consolidação em 2012 como um processo científico reconhecido para o mapeamento de um tema que permite atender aos objetivos específicos do pesquisador (TASCA et al., 2010; LACERDA; ENSSLIN; ENSSLIN, 2012).

O ProKnow- $C$ é composto por 4 macro etapas, conforme demonstrado na figura 1. Para esta pesquisa, foram realizadas as duas primeiras etapas, seleção de portfólio bibliográfico e bibliometria, descritas na seção 4, a qual aborda os resultados e discussões para o tema "avaliação de produção científica" (ENSSLIN et al., 2010; TASCA et al., 2010; BORTOLUZZI et al., 2011; LACERDA; ENSSLIN; ENSSLIN, 2011; ENSSLIN; ENSSLIN; PACHECO, 2012; BRUNA et al., 2012; ROSA et al., 2012). 


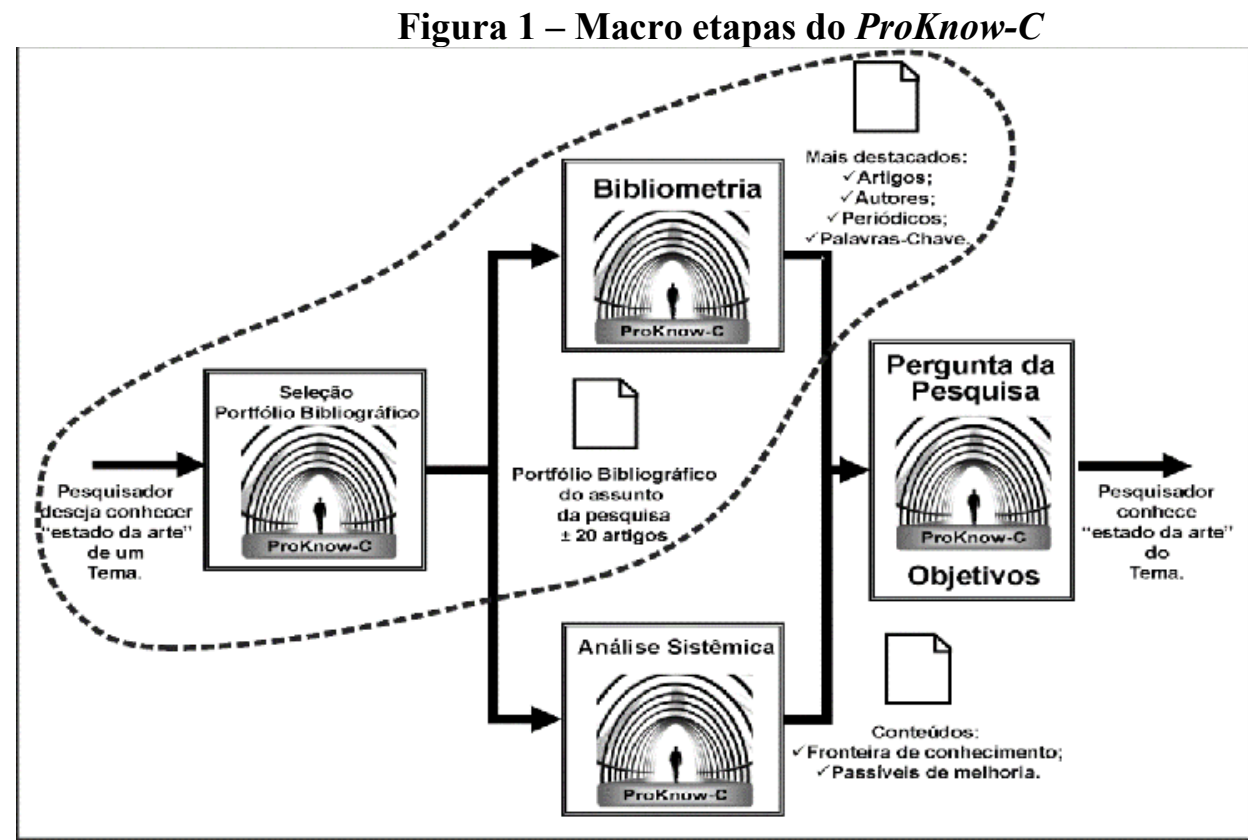

Fonte: Tasca et al. (2010), Lacerda, Ensslin e Ensslin (2011) e Bruna et al. (2012).

Contudo, ressalta-se a conceituação das seguintes etapas:

a) Portfólio Bibliográfico: conjunto restrito de publicações com reconhecimento e destaque científico, com Título, Resumo e Conteúdo Completo, alinhados com um determinado tema segundo a percepção e delimitação de um pesquisador (TASCA et al., 2010; BORTOLUZZI et al., 2011; LACERDA; ENSSLIN; ENSSLIN, 2011; AZEVEDO et al., 2011).

b) Bibliometria: processo de evidenciação quantitativa dos dados estatísticos de um conjunto definido de artigos (Portfólio Bibliográfico) para a gestão da informação e do conhecimento científico de um dado assunto, realizado por meio da contagem de documentos (TASCA et al., 2010; BORTOLUZZI et al., 2011; LACERDA; ENSSLIN; ENSSLIN, 2011; ROSA et al., 2011).

Diferentemente de métodos como os abordados na "Systematic Literature Review" (KITCHENHAM et al., 2009) e na "Systematic Review" (PITTAWAY et al., 2004), o ProKnow-C auxilia na construção de um Portfólio Bibliográfico em forma de fluxograma e descritivo, simultaneamente, conforme mostra a Figura 2, na qual é ilustrada a etapa de seleção de artigos para o Portfólio Bibliográfico.

A aplicabilidade desse processo pode ser conferida em pesquisas similares que abordaram assuntos distintos e obtiveram sucesso na construção do conhecimento sobre um tema até então pouco conhecido para os pesquisadores em questão, como é o caso de Lacerda, Ensslin e Ensslin (2011), Afonso et al. (2012), Chaves et al. (2012), Ensslin, Ensslin e Pacheco (2012), entre outros. 


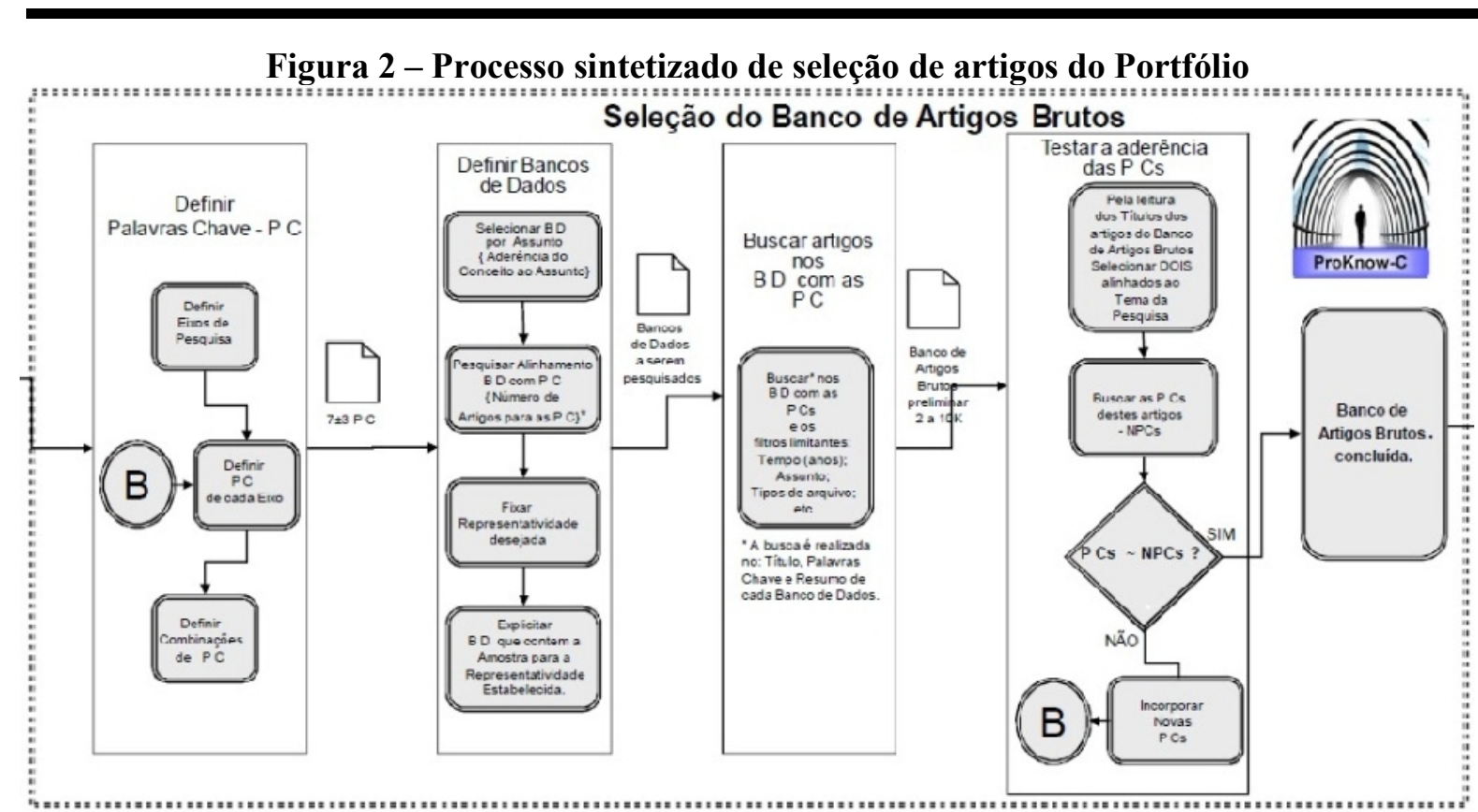

Fonte: Adaptado de Lacerda, Ensslin e Ensslin (2012).

\section{Referencial Teórico}

O objetivo desta seção é apresentar abordagens relevantes para dar suporte à avaliação de produção científica a partir dos artigos selecionados, a fim de compor o portfólio bibliográfico.

Essa avaliação tem recebido atenção especial frente às inúmeras opções de bases de dados online disponíveis que indexam, em escala mundial, as publicações científicas, além da grande quantidade de pesquisas e pesquisadores que nelas se encontram, as quais oferecem um volume de informações sem precedentes na história da ciência.

Embora haja diversas métricas, como, por exemplo, os tradicionais fatores de impacto de revistas científicas e a revisão de artigos por pares de especialistas, que muitos consideram ser mais relevantes para demonstrar a qualidade das publicações (JIN et al., 2007; BUTLER, 2008), ainda não há um consenso de quais dados devam ser levados ou não em consideração na avaliação de artigos científicos.

Porém, Harnad (2008) chama a atenção para o fato de que avaliar os pesquisadores utilizando apenas os fatores de impacto das revistas em que publicam é comparável a avaliar alunos pela reputação da universidade e não pelas notas obtidas por eles. Por outro lado, van Raan (2005) defende a ideia da avaliação por pares em conjunto com indicadores de desempenho.

Segundo Coccia (2004), Costas et al. (2010) e Hall (2011), o desempenho dos pesquisadores individuais pode ser analisado pelas perspectivas bibliométricas, tanto para monitorar o desenvolvimento científico quanto tecnológico. Os indicadores bibliométricos se tornaram um fator chave como suporte para entender e gerenciar as políticas de decisões, agências de fomento, influências da idade, gênero, colaborações científicas, decisões de pares de especialistas, entre outros objetivos (VAN RAAN, 2005; VAN RAAN, 2006; COSTAS et al., 2010). 
Hirsch (2005) propôs analisar os pesquisadores por meio de um indicador intitulado de $h$-index. Esse índice, em sua simplicidade, utiliza o número de $h$ artigos que receberam, cada um deles, pelo menos, $h$ citações. Como resultado, o autor recebe um índice $h$ que reflete o seu desempenho científico.

Após essa proposta, foram desenvolvidas outras abordagens para atender parâmetros que o $h$-index desconsidera. O índice $h$ sucessivo (SCHUBERT, 2007 apud ARENCIBIAJORGE et al., 2008) considera o desempenho dos pesquisadores individuais e, após isso, analisa hierarquicamente o departamento e a universidade.

Os índices g-index, A-index, R-index e AR-index (JIN et al., 2007) utilizam a soma das citações dos artigos em seus cálculos e/ou os anos de carreira do pesquisador. Neste último, o valor do índice poderá diminuir caso a produção científica também diminua, fato que não ocorre com o h-index.

Outra variação do próprio índice $h$ é o $h_{I}$, proposto por Batista et al. (2006). Esse índice tem por objetivo analisar pesquisadores de diferentes áreas levando em conta a média do número de autores de cada artigo publicado.

Uma das questões, porém, em que há mais consonância na comunidade científica é a necessidade de avaliar os pesquisadores dentro de suas áreas de atuação. Hirsch (2005), Meho e Spurgin (2005) e Batista et al. (2006) frisam que devem ser utilizados métodos, ferramentas e indicadores que sejam justapostos com as especificidades de cada campo, não cabendo a avaliação de seus desempenhos comparados entre diferentes áreas, quando utilizados indicadores que são robustos e generalizados.

Informações como idade, gênero, livros, capítulos de livros, congressos, revisões, editor, organizador de seção e autocitações também são questões de debate sobre a inclusão ou não no conjunto avaliativo (COCCIA, 2004; MEHO; SPURGIN, 2005; BUTLER, 2008; ONDER et al., 2008; HALL, 2011; KOZAK; BORNMANN, 2012).

Por fim, estudos na Turquia (ONDER et al., 2008), Austrália (BUTLER, 2008) e China (LI et al., 2012) têm demonstrado que a utilização de um conjunto de indicadores podem trazer resultados representativos na avaliação e promover o desenvolvimento científico qualitativo, qualidade esta que, segundo alguns autores (VAN RAAN, 2006; HARNAD, 2008; COSTAS et al., 2010; HALL, 2011), vem sendo substituída por números ao utilizar apenas indicadores quantitativos.

\section{Resultados e Discussões}

Nesta seção, apresentam-se os resultados da pesquisa e a seleção de artigos utilizando o ProKnow-C para o contexto de avaliação de produção científica, a fim de (a) compor um portfólio bibliográfico e (b) realizar a análise bibliométrica do portfólio bibliográfico.

\subsection{Portfólio Bibliográfico (Pb)}

Para a seleção do Portfólio Bibliográfico - PB -, realizaram-se as seguintes fases: 1) seleção do banco bruto de artigos; 2) filtro quanto à redundância, título e reconhecimento científico; 3) filtro do banco bruto de artigos não repetidos e com títulos alinhados; e 4) filtro quanto ao alinhamento integral do artigo (TASCA et al., 2010; LACERDA; ENSSLIN; ENSSLIN, 2011; BORTOLUZZI et al., 2011; ROSA et al., 2012).

103 ISSN 2175-8069, UFSC, Florianópolis, v. 10, n. 20, p. 97-112, mai./ago. 2013 
2013.

Tais fases estão previstas no ProKnow-C e foram realizadas no mês de janeiro de

1) Para iniciar o processo o ProKnow-C, é requerido que sejam definidos os eixos da pesquisa. Assim, dois eixos de pesquisa foram definidos: avaliação de desempenho e produção científica. Para cada eixo, foram definidas cinco palavras-chave, sendo elas: Performance Measurement, Performance Evaluation, Performance Appraisal, Performance Assessment e Performance Management, para o eixo Avaliação de Desempenho; Scientific Production, Scientific Research, Scientometric, Scientist, e Publication, para o eixo Produção Científica. Cada palavra-chave (PC) foi combinada com as PC do eixo oposto, o que resultou em 25 combinações. Essas combinações foram utilizadas para pesquisar em seis bases de dados selecionadas no portal Periódicos da CAPES, a saber: "Web of Science", "Scopus", "Ebsco", "Springer", "Wiley", e "ProQuest". Esta pesquisa resultou, dessa forma, no levantamento de 2.514 artigos publicados em periódicos internacionais entre janeiro de 2000 e janeiro de 2013.

2) Na fase 2 (filtro quanto à redundância, título e reconhecimento científico), foram importados para o gerenciador bibliográfico Endnote X5 os artigos encontrados na pesquisa, onde foi realizada a exclusão de 388 artigos duplicados.

3) $\mathrm{Na}$ terceira fase (filtro do banco bruto de artigos não repetidos e com títulos alinhados), foi realizada a leitura de títulos dos 2.126 artigos para identificar aqueles que atendiam aos critérios dos pesquisadores, o que resultou no levantamento de 62 artigos com títulos alinhados ao tema desta pesquisa. No Google Acadêmico, buscouse o número de citações dos 62 artigos. Desses, 30 publicações representaram $95 \%$ do total de citações, pois obtiveram 10 citações ou mais, as quais foram submetidas à leitura dos resumos, sendo selecionados 14 artigos com resumos alinhados. Outro conjunto de 15 artigos (dos que não figuravam dentre os mais citados) passou por uma reanálise por serem artigos publicados há menos de dois anos, os quais não tiveram tempo suficiente para serem citados. Desses, 02 artigos estavam com os resumos alinhados ao tema.

4) $\mathrm{Na}$ fase 4 (filtro quanto ao alinhamento integral do artigo), o conjunto de 16 artigos (14 mais citados e com resumo alinhado e 02 da reanálise) foi submetido à leitura integral. Ao final, consideraram-se 12 artigos alinhados ao tema.

Adicionalmente, analisaram-se e se relacionaram as referências dos artigos selecionados para realização do teste de representatividade. Totalizaram-se 145 referências publicadas a partir de 2000 , entre as quais 50 representaram $80 \%$ do número de citações. Dessas referências, 03 foram incorporadas aos 12 artigos selecionados anteriormente, por estarem alinhadas integralmente ao tema desta pesquisa.

Assim, 15 artigos (disponibilizados de forma gratuita) compõem o Portfólio Bibliográfico, os quais serviram de base para o referencial teórico e a bibliometria deste trabalho, conforme mostra a Tabela 1. 
Avaliação de produção científica de pesquisadores: mapeamento das publicações científicas

Tabela 1 - Portfólio Bibliográfico

\begin{tabular}{|c|c|c|c|c|}
\hline$\#$ & Autor & Artigo & Periódico & Ano \\
\hline 1 & COCCIA, M. & $\begin{array}{l}\text { New models for measuring the R\&D } \\
\text { performance and identifying the } \\
\text { productivity of public research institutes }\end{array}$ & R\&D Management & 2004 \\
\hline 2 & HIRSCH, J. E. & $\begin{array}{l}\text { An index to quantify an individual's } \\
\text { scientific research output }\end{array}$ & $\begin{array}{l}\text { Proceedings of the National } \\
\text { Academy of Sciences of the } \\
\text { United States of America }\end{array}$ & 2005 \\
\hline 3 & $\begin{array}{l}\text { MEHO, L. I.; } \\
\text { SPURGIN, K. M. }\end{array}$ & $\begin{array}{l}\text { Ranking the research productivity of } \\
\text { library and information science faculty } \\
\text { and schools: an evaluation of data sources } \\
\text { and research methods }\end{array}$ & $\begin{array}{l}\text { Journal of the American } \\
\text { Society for Information } \\
\text { Science and Technology }\end{array}$ & 2005 \\
\hline 4 & $\begin{array}{l}\text { VAN RAAN, A. } \\
\text { P. J. }\end{array}$ & $\begin{array}{l}\text { Fatal attraction: conceptual and } \\
\text { methodological problems in the ranking of } \\
\text { universities by bibliometric methods }\end{array}$ & Scientometrics & 2005 \\
\hline 5 & $\begin{array}{l}\text { BATISTA, P. D. } \\
\text { et al. }\end{array}$ & $\begin{array}{l}\text { Is it possible to compare researchers with } \\
\text { different scientific interests? }\end{array}$ & Scientometrics & 2006 \\
\hline 6 & $\begin{array}{l}\text { VAN RAAN, A. } \\
\text { P. J. }\end{array}$ & $\begin{array}{l}\text { Comparison of the Hirsch-index with } \\
\text { standard bibliometric indicators and with } \\
\text { peer judgment for } 147 \text { chemistry research } \\
\text { groups }\end{array}$ & Scientometrics & 2006 \\
\hline 7 & JIN, B. H. et al. & $\begin{array}{l}\text { The R- and AR-indices: Complementing } \\
\text { the h-index }\end{array}$ & Chinese Science Bulletin & 2007 \\
\hline 8 & ONDER, C. et al. & $\begin{array}{l}\text { Institutional change and scientific } \\
\text { research: a preliminary bibliometric } \\
\text { analysis of institutional influences on } \\
\text { Turkey's recent social science } \\
\text { publications }\end{array}$ & Scientometrics & 2008 \\
\hline 9 & BUTLER, L. & $\begin{array}{l}\text { Using a balanced approach to } \\
\text { bibliometrics: quantitative performance } \\
\text { measures in the Australian Research } \\
\text { Quality Framework }\end{array}$ & $\begin{array}{l}\text { Ethics in Science and } \\
\text { Environmental Politics }\end{array}$ & 2008 \\
\hline 10 & $\begin{array}{l}\text { ARENCIBIA- } \\
\text { JORGE, R. et al. }\end{array}$ & $\begin{array}{l}\text { Applying successive } \mathrm{H} \text { indices in the } \\
\text { institutional evaluation: A case study }\end{array}$ & $\begin{array}{l}\text { Journal of the American } \\
\text { Society for Information ... }\end{array}$ & 2008 \\
\hline 11 & HARNAD, S. & $\begin{array}{l}\text { Validating research performance metrics } \\
\text { against peer rankings }\end{array}$ & $\begin{array}{l}\text { Ethics in Science and } \\
\text { Environmental Politics }\end{array}$ & 2008 \\
\hline 12 & $\begin{array}{l}\text { COSTAS, R. et } \\
\text { al. }\end{array}$ & $\begin{array}{l}\text { Bibliometric classificatory approach for } \\
\text { the study and assessment of research } \\
\text { performance at the individual level: the } \\
\text { effects of age on productivity and impact }\end{array}$ & $\begin{array}{l}\text { Journal of the American } \\
\text { Society for Information ... }\end{array}$ & 2010 \\
\hline 13 & HALL, C. M. & $\begin{array}{l}\text { Publish and perish? Bibliometric analysis, } \\
\text { journal ranking and the assessment of } \\
\text { research quality in tourism }\end{array}$ & Tourism Management & 2011 \\
\hline 14 & LI, F. et al. & $\begin{array}{l}\text { Performance evaluation of research } \\
\text { universities in Mainland China, Hong } \\
\text { Kong and Taiwan: based on a two- } \\
\text { dimensional approach }\end{array}$ & Scientometrics & 2012 \\
\hline 15 & $\begin{array}{l}\text { KOZAK, M.; } \\
\text { BORNMANN, L. }\end{array}$ & $\begin{array}{l}\text { A New Family of Cumulative Indexes for } \\
\text { Measuring Scientific Performance }\end{array}$ & PLoS ONE & 2012 \\
\hline
\end{tabular}

Fonte: Dados da pesquisa.

105 ISSN 2175-8069, UFSC, Florianópolis, v. 10, n. 20, p. 97-112, mai./ago. 2013 
Figura 3 - ProKnow-C: origem dos dados bibliométricos

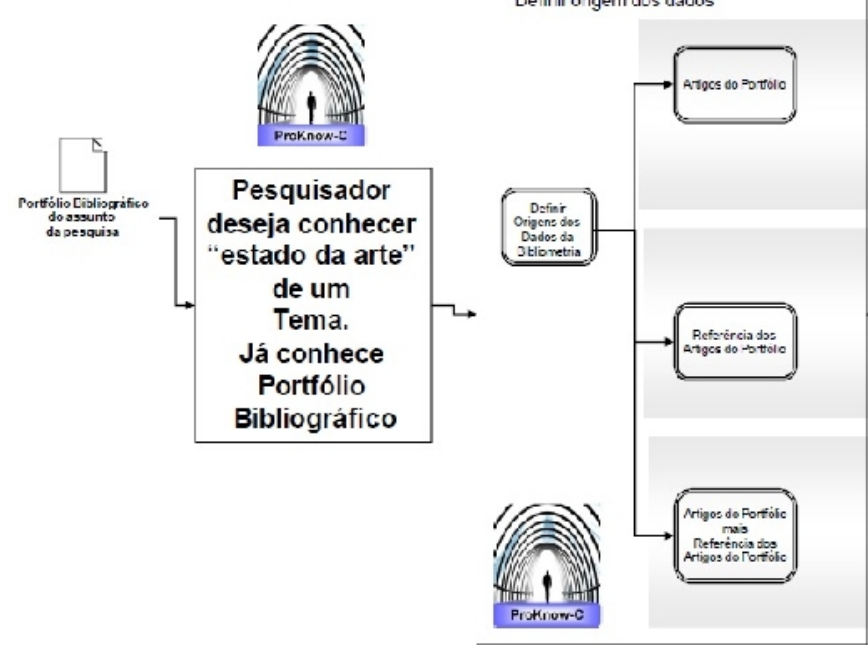

Fonte: adaptado de Lacerda, Ensslin e Ensslin (2012).

\subsection{Análise Bibliométrica}

Nessa etapa, para realizar a bibliometria, o ProKnow-C utilizou, como fonte de origem dos dados, os artigos do $\mathrm{PB}$, os periódicos do $\mathrm{PB}$ em conjunto com suas referências, os autores do PB, e as palavras-chave do PB (Figura 3). Para efeito desta publicação, as demais análises não serão contempladas.

Para a análise bibliométrica, quatro aspectos foram considerados: (a) Periódicos de Destaque; (b) Artigos de Destaque; (c) Autores de Destaque; e, (d) Palavras-chave de Destaque (TASCA et al., 2010; LACERDA; ENSSLIN; ENSSLIN, 2011; BORTOLUZZI et al., 2011; ROSA et al., 2012).

No primeiro aspecto, Periódicos de Destaque, analisou-se a relevância dos periódicos tanto no PB quanto nas referências, conforme mostra a Figura 4.

Figura 4 - Periódicos de Destaque

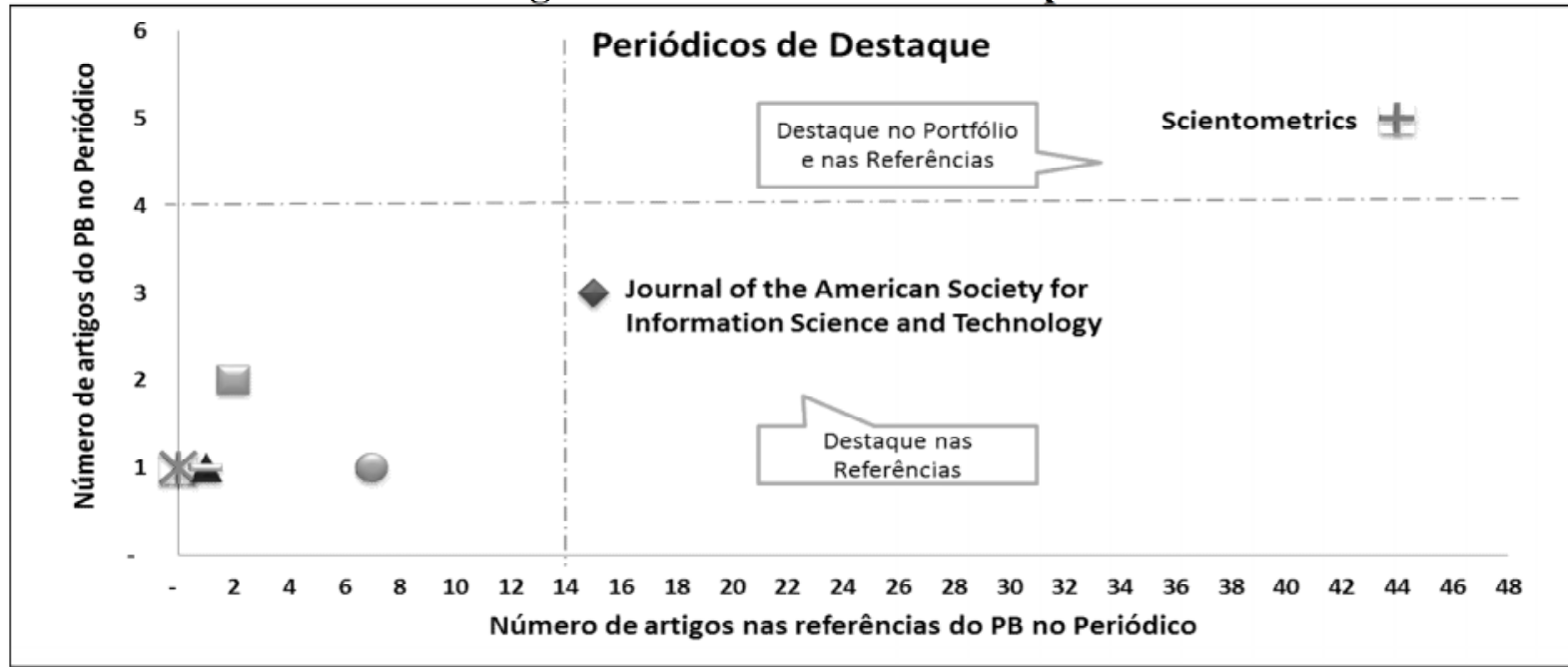

Fonte: Dados da pesquisa. 
O quadrante superior direito apresenta o "Scientometrics" como o periódico com destaque no PB e nas referências. Ele contribui com 44 artigos nas referências e 05 artigos no PB. Apresenta um fator de impacto, segundo o JCR, de 1,966, e o SJR, de 1,384, com um total de 4.457 citações. O "Journal of the American Society for Information Science and Technology" apresenta destaque nas referências, com 1.481 citações, enquanto que o JCR, de 2,981, e o SJR, de 1,517.

$\mathrm{O}$ aspecto Artigos de Destaque foi analisado conforme a Figura 5. O artigo "An index to quantify an individual's scientific research output", de Hirsch (2005), apresenta destaque tanto no PB como nas referências. Ao propor um indicador de fácil utilização ( $h$-index), ganhou destaque na comunidade científica, recebendo 3.065 citações, segundo o Google Acadêmico, e 05 citações nas referências do PB.

Figura 5 - Artigos de Destaque

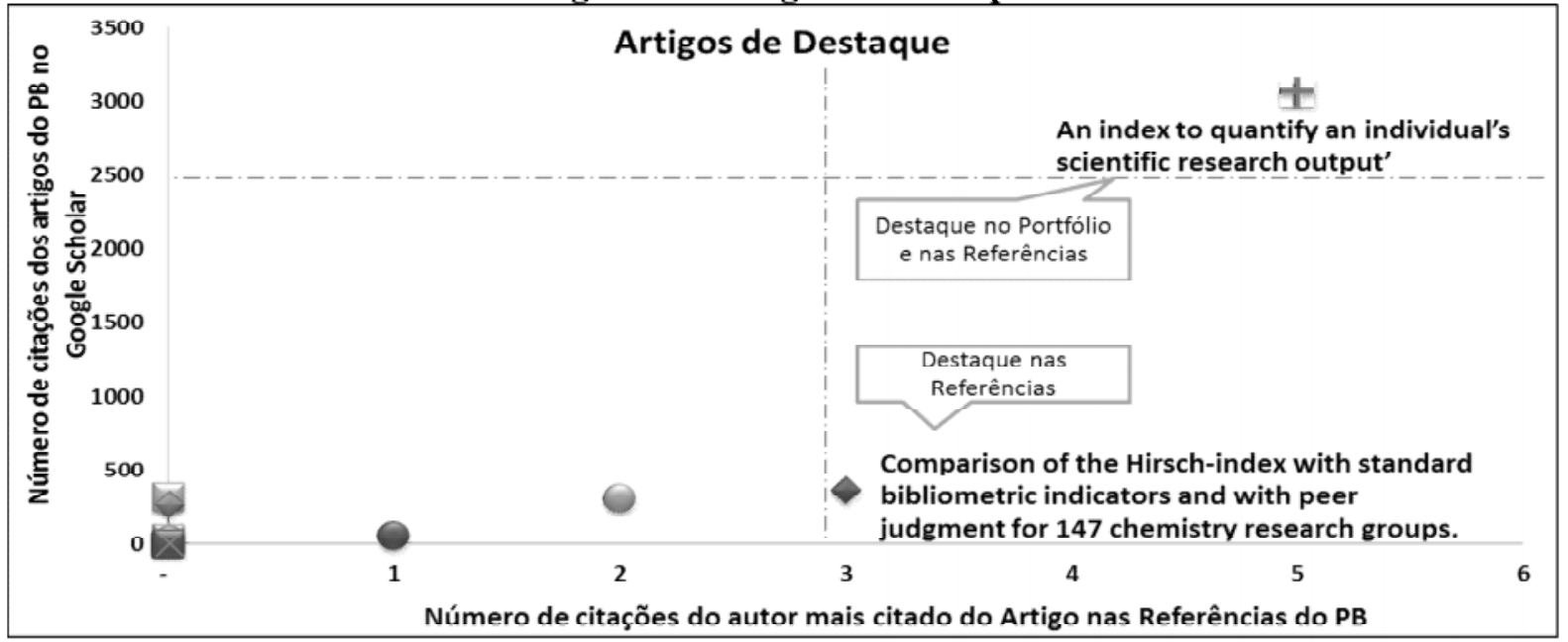

Fonte: Dados da pesquisa.

Como destaque nas referências, tem-se o artigo "Comparison of the Hirsch-index with standard bibliometric indicators and with peer judgment for 147 chemistry research groups" de van Raan (2006), no qual demonstra a correlação entre o h-index, os indicadores bibliométricos e a avaliação por pares de especialistas, sendo citado 03 vezes nas referências do PB e possuindo 366 citações.

A Figura 6, a seguir, evidencia os autores que mais contribuem com artigos relevantes no Portfólio Bibliográfico e nas referências. São 33 autores para os 15 artigos do PB. O destaque no PB e nas referências é o autor van Raan, com 02 artigos no Portfólio Bibliográfico e 08 artigos nas referências do $\mathrm{PB}$, sendo apenas 01 autocitação. Os demais autores contribuem com um artigo cada para o PB, alguns deles como coautores. Cabe destacar os autores Bornmann, com 08 trabalhos listados nas referências, sendo, desses, 06 são autocitações. Van Leeuwen. por sua vez, apresenta 05 artigos nas referências do PB, sendo 03 autocitações, além de Bordons e Costas, cada um com 05 artigos nas referências do PB, sendo eles autocitações. 
Figura 6 - Autores de Destaque

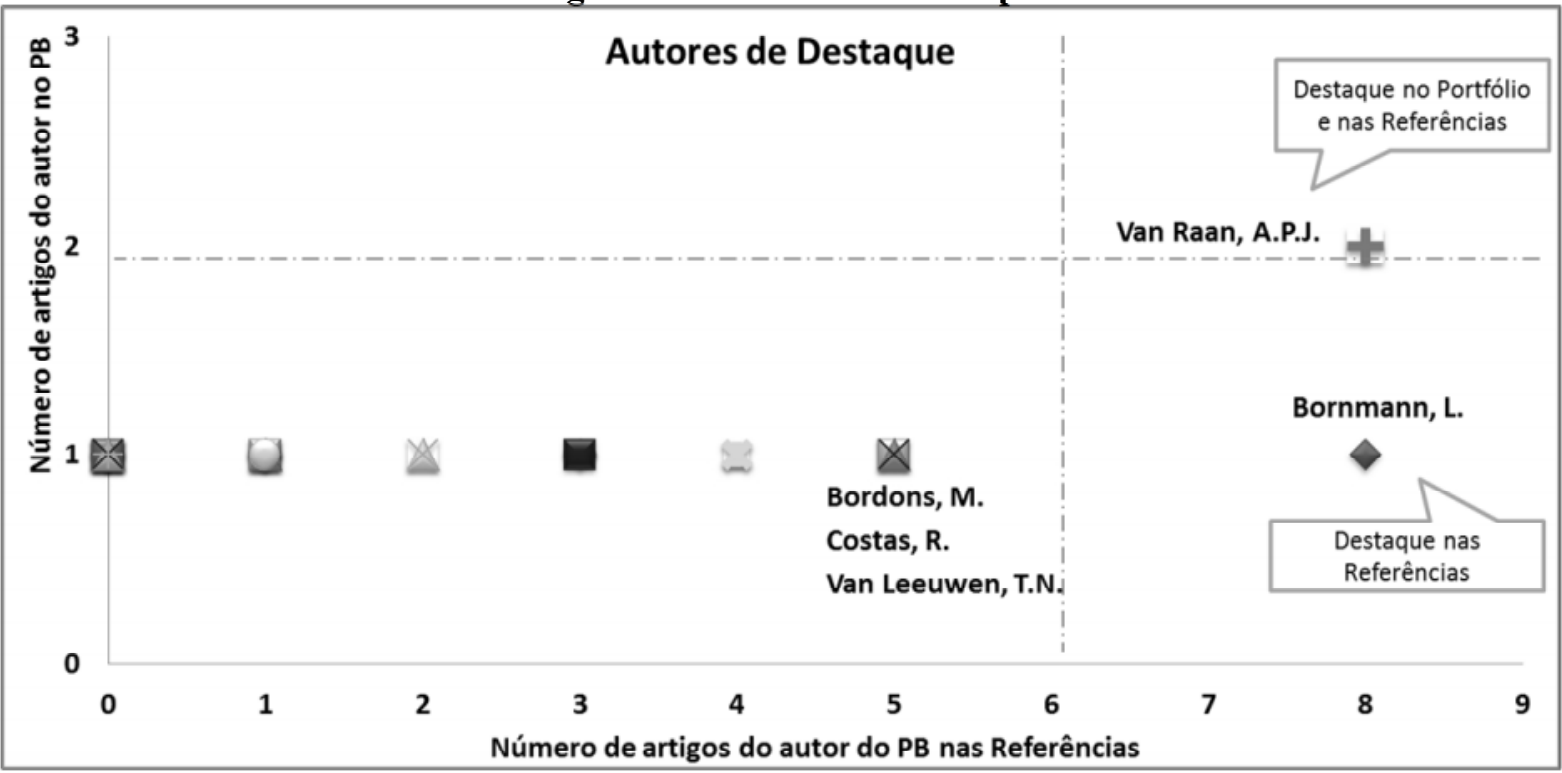

Fonte: Dados da pesquisa.

O exposto na figura 7 evidencia as Palavras-chave de Destaque do Portfólio Bibliográfico. Os termos "Bibliometrics", "Citation" $e$ "Research Assessment" se repetem duas vezes no conjunto exposto de 32 palavras-chave - as demais aparecem uma vez.

Figura 7 - Palavras-chave de destaque

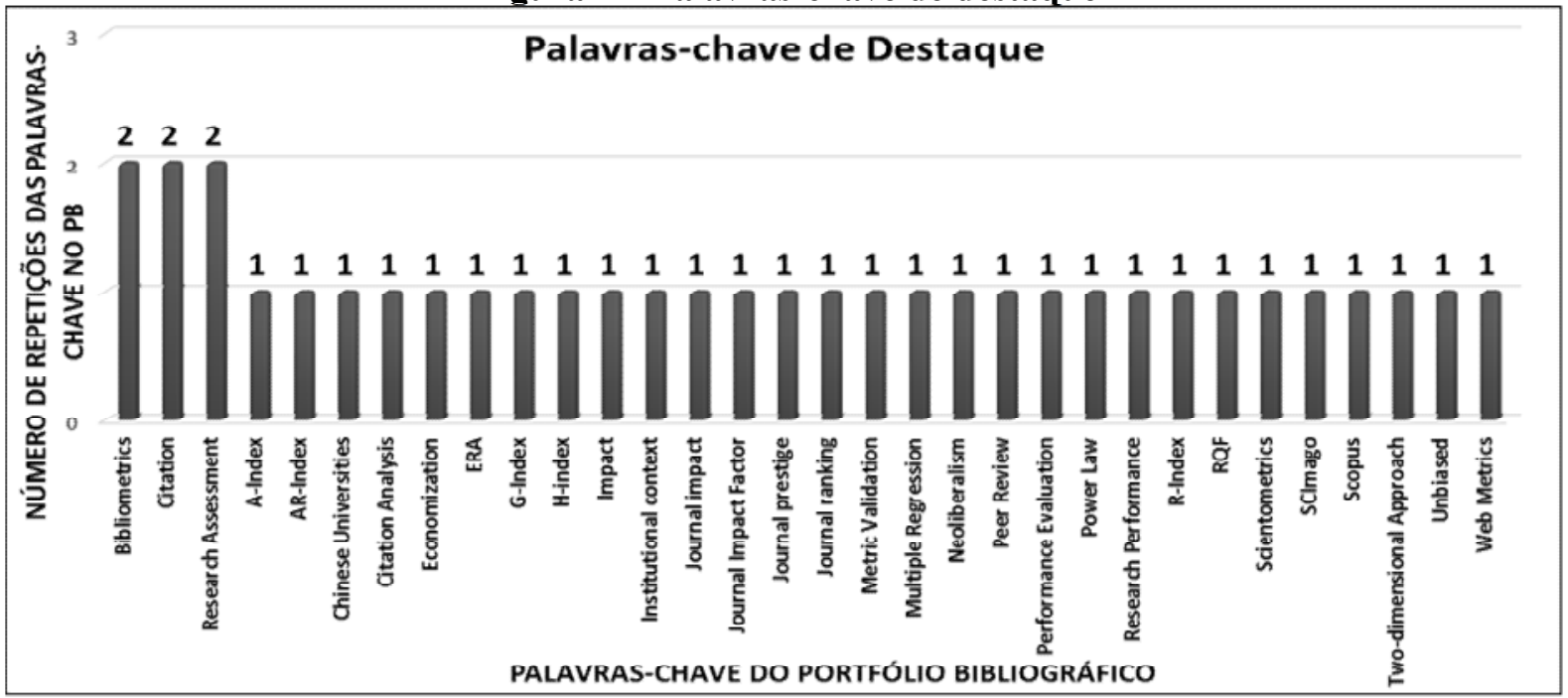

Fonte: Dados da pesquisa.

Pode-se, portanto, entender que se trata de um assunto que possui uma dispersão de conhecimento, sendo que, nesta pesquisa, não ocorreram palavras-chave usadas com frequência ao referido tema. 


\section{Considerações Finais}

A busca pela inovação e atualização levou a comunidade acadêmica e empresarial internacional, quando da avaliação de seus profissionais, a levar em conta sua produção científica. Isso acontece porque as produções científicas são meios para tornar público pesquisas e descobertas relevantes e transmitir à comunidade científica e à sociedade o conhecimento gerado. Porém, dado o volume de informações, abordagens e métodos disponíveis em bancos de dados científicos, surgiu o problema de como evidenciar as publicações e suas respectivas variáveis de destaque sobre o tema "Avaliação da Produção Científica de Pesquisadores".

Para atender ao objetivo central desta pesquisa, identificaram-se os artigos que mais contribuíram, conforme as delimitações postas pelos pesquisadores, para que se compreendesse como é abordado o tema "Avaliação da Produção Científica de Pesquisadores", disponível na literatura internacional. Como delimitações da pesquisa destaca-se que a mesma se restringiu à coleta de dados e artigos publicados em periódicos internacionais entre janeiro de 2000 e janeiro de 2013, disponíveis gratuitamente em bancos de dados da CAPES, e que estivessem alinhados ao tema proposto.

O ProKnow-C foi utilizado como instrumento de intervenção por disponibilizar um processo estruturado que permitiu: (a) selecionar um portfólio bibliográfico de 15 artigos com reconhecimento científico e alinhado ao tema, segundo as percepções do pesquisador; e (b) realizar a análise bibliométrica no portfólio que apontou o "Scientometrics" como periódico de destaque, o autor van Raan como o autor com o mais alto número de trabalhos, o artigo "An index to quantify an individual's scientific research output" como o mais citado, e os termos Bibliometrics, Citation, e Research Assessment entre as palavras-chave de destaque.

Para pesquisas futuras, sugere-se realizar as duas últimas etapas do ProKnow-C: (iii) análise sistêmica e (iv) definição da pergunta de pesquisa e objetivo de pesquisa, uma vez que oferece oportunidades de encontrar lacunas na literatura para futuras pesquisas.

\section{Referências}

AFONSO, M. H. F. et al. Como construir conhecimento sobre o tema de pesquisa? Aplicação do processo Proknow- $C$ na busca de literatura sobre avaliação do desenvolvimento sustentável. Revista de Gestão Social e Ambiental, v. 5, p. 47-62. 2012.

ARENCIBIA-JORGE, R. et al. Applying successive h indices in the institutional evaluation: a case study. Journal of the American Society for Information Science and Technology, v. 59, n. 1, p. 155-157. 2008.

AZEVEDO, R. et al. Avaliação de desempenho do processo de orçamento: estudo de caso em uma obra de Construção Civil. Ambiente Construído (Online), v. 11, p. 85-104. 2011.

BATISTA, P. D. et al. Is it possible to compare researchers with different scientific interests? Scientometrics, v. 68, n. 1, p. 179-189. 2006. 
BORTOLUZZI, S. C. et al. A avaliação de desempenho em redes de pequenas e médias empresas: estado da arte para as delimitações postas pelo pesquisador. Revista Eletrônica de Estratégia \& Negócios, v. 4, n. 2, p. 202-222. 2011.

BRUNA, E. D. Jr. et al. Seleção e análise de um portfólio de artigos sobre avaliação de desempenho na cadeia de suprimentos. Gestão da Produção, Operações e Sistemas, v. 7, n. 1, p. 113-125. 2012.

BUTLER, L. Using a balanced approach to bibliometrics: quantitative performance measures in the Australian Research Quality Framework. Ethics in Science and Environmental Politics, v. 8, n. 2, p. 1-10. 2008.

CALAZANS, A. T. S. Qualidade da informação: conceitos e aplicações. TransInformação, v. 20, n. 1, p. 29-45. 2008.

CHAVES, L. C. et al. Mapeamento do tema gestão do apoio à decisão quando analisado sob a ótica de seus resultados. Sistema \& Gestão, v. 7, n. 3, p. 336-348. 2012.

COCCIA, M. New models for measuring the R\&D performance and identifying the productivity of public research institutes. R\&D Management, v. 34, n. 3, p. 267-280. 2004.

COSTAS, R. et al. Bibliometric classificatory approach for the study and assessment of research performance at the individual level: the effects of age on productivity and impact. Journal of the American Society for Information Science and Technology, v. 61, n. 8, p. 1564-1581. 2010.

ENSSLIN, L. et al. ProKnow-C, Knowledge Development Process-Constructivist. Processo técnico com patente de registro pendente junto ao INPI, Brasil, 2010.

ENSSLIN, L.; ENSSLIN, S. R.; PACHECO, G. C. Um estudo sobre segurança em estádios de futebol baseado na análise da literatura internacional. Perspectivas em Ciências da Informação, v. 17, n. 2, p. 71-91. 2012.

FERREIRA, N. S; YOSHIDA, E. M. P. Produção Científica sobre psicoterapias breves no Brasil e demais países latino-americanos (1990-2000). Estudos de Psicologia, v. 3, n. 3, p. 523-531. 2004.

GIL, A. C. Métodos e técnicas de pesquisa social. 6. ed. São Paulo: Atlas, 2008.

HALL, C. M. Publish and perish? Bibliometric analysis, journal ranking and the assessment of research quality in tourism. Tourism Management, v. 32, n. 1, p. 16-27. 2011.

HARNAD, S. Validating research performance metrics against peer rankings. Ethics in Science and Environmental Politics, v. 8, n. 1, p. 1-10. 2008. 
HIRSCH, J. E. An index to quantify an individual's scientific research output. Proceedings of the National Academy of Sciences of the United States of America, v. 102, n. 46, p. 16569-16572. 2005.

JIN, B. H. et al. The r- and ar- indices: complementing the h-index. Chinese Science Bulletin, v. 52, n. 6, p. 855-863. 2007.

KITCHENHAM, B. et al. Systematic literature reviews in software engineering. Information and Software Technology, v. 51, n. 1, p. 7-15. 2009.

KOBASHI, Y.; SANTOS, R. N. M. Institucionalização da pesquisa científica no Brasil: cartografia temática e de redes sociais por meio de técnicas bibliométricas.

TransInformação, v. 18, n. 1, p. 27-36. 2006.

KOZAK, M.; BORNMANN, L. A new family of cumulative indexes for measuring scientific performance. Plos One, v. 7, n. 10, p. 1-4. 2012.

LACERDA, R. T. O.; ENSSLIN, L.; ENSSLIN, S. R. A performance measurement framework in portfolio management: a constructivist case. Management Decision, v. 49, p. 1-15. 2011.

LACERDA, R. T. O.; ENSSLIN, L.; ENSSLIN, S. R. Uma análise bibliométrica da literatura sobre estratégia e avaliação de desempenho. Gestão \& Produção, v. 19, n. 1, p. 59-78. 2012.

LI, F. et al. Performance evaluation of research universities in Mainland China, Hong Kong and Taiwan: based on a two-dimensional approach. Scientometrics, v. 90, n. 1, p. 531-542. 2012.

MEHO, L. I.; SPURGIN, K. M. Ranking the research productivity of library and information science faculty and schools: an evaluation of data sources and research methods. Journal of the American Society for Information Science and Technology, v. 56, n. 12, p. 13141331. 2005.

ONDER, C. et al. Institutional change and scientific research: a preliminary bibliometric analysis of institutional influences on Turkey's recent social science publications.

Scientometrics, v. 76, n. 3, p. 543-560. 2008.

PITTAWAY, L. et al. Networking and innovation: a systematic review of the evidence. International Journal of Management Reviews, v. 5-6, n. 3-4, p. 137-168. 2004.

RICHARDSON, R. J. Pesquisa social: métodos e técnicas. 3. ed. São Paulo: Atlas, 2008.

ROSA, F. S. et al. Evidenciação ambiental para gestão interna: um estudo sobre as potencialidades e oportunidades do tema. Engenharia Sanitária e Ambiental, v. 16, p. 157166. 2011.

111 ISSN 2175-8069, UFSC, Florianópolis, v. 10, n. 20, p. 97-112, mai./ago. 2013 
ROSA, F. S. et al. Management environmental disclosure: a constructivist case. Management Decision, v. 50, n. 6, p. 1117-1136. 2012.

TASCA, J. E. et al. An approach for selecting a theoretical framework for the evaluation of training programs. Journal of European Industrial Training, v. 34, n. 7, p. 631-655. 2010.

VAN RAAN, A. F. J. Comparison of the Hirsch-index with standard bibliometric indicators and with peer judgment for 147 chemistry research groups. Scientometrics, v. 67, n. 3, p. 491-502. 2006.

VAN RAAN, A. F. J. Fatal attraction: conceptual and methodological problems in the ranking of universities by bibliometric methods. Scientometric, v. 62, n. 1, p. 133-143. 2005. 